\title{
GPM1 - a catalog of absolute proper motions of stars with respect to galaxies
}

\author{
S.P. Rybka and A.I. Yatsenko \\ Main Astronomical Observatory of National Academy of Sciences, Goloseevo, DSP-650, Kiev-22, Ukraine
}

Received 4 July, 1995; accepted 14 March, 1996

\begin{abstract}
The description of the first version of the General Compiled Catalogue of Absolute Proper Motions $(\text { GPM1 })^{1}$ for a sample of HIPPARCOS stars, derived with respect to galaxies within the plan called Catalogue of Faint Stars (KSZ, Deutch 1952), is presented. The principal aim of the GPM1 construction was to provide absolute proper motions of stars to determine the rotation of the HIPPARCOS system. The GPM1 catalogue contains 977 HIPPARCOS Input Catalogue stars with $V$ magnitudes $5^{\mathrm{m}}-11^{\mathrm{m}}$ in 180 fields north of -25 degrees of declination. The accuracy of the proper motions is $8 \mathrm{mas} / \mathrm{yr}$ (milliarcseconds per year). Comparison of proper motions of GPM1 with those of the PPM and ACRS was performed and analyzed with respect to systematic errors caused by spurious rotation of the FK5 system. The standard errors show that the rotation may be determined with an accuracy better than $1 \mathrm{mas} / \mathrm{yr}$.
\end{abstract}

Key words: astrometry — reference systems

\section{Introduction}

The system determined from the observations carried out by the ESA satellite HIPPARCOS provides an exclusively precise catalogue. However, the highly accurate HIPPARCOS reference frame needs a link to an inertial coordinate system. There are some methods for the link of the HIPPARCOS data using ground based observations. One of them deals with the use of absolute proper motion of stars determined with respect to galaxies by means of photographic astrometry. Preliminary results have shown that this method is competitive with other more expensive techniques (Yatsenko et al. 1987).

Proper motion programmes that may be used for the link are in progress at several observatories (Lick,

Send offprint requests to: S.P. Rybka

1 Catalog is only available in electronic form at the CDS via anonymous ftp cdsarc.u-strasbg.fr or ftp 130.79.128.5.
U.S.A.; Hamburg, Bonn and Potsdam, Germany; Kiev, Ukraine). The Kiev part is based on the application of absolute proper motions of stars obtained from photographic observations on the programmes called Catalogue of Faint Stars (KSZ, Deutch 1952) and Study of the Main Meridional Section of the Galaxy (MEGA, Kharchenko 1987). The present paper is devoted to the description of the new version of the compiled catalogue performed within the KSZ programme.

\section{The proper motion material}

The original KSZ plan of photography of selected sky fields with galaxies involves 205 areas north of -25 degrees of declination. The programme was proposed for several observatories of the former Soviet Union, China, Rumania and Spain. There are some resulting individual catalogues of absolute proper motions of stars compiled at five observatories (Kiev, Pulkovo, Moscow, Tashkent and Shanghai) according to the cooperative programme KSZ. The ultimate goal is to combine available catalogues in General Proper Motion catalog (GPM). At present, the compilation of the GPM catalog of stars referred to galaxies is under work at Main Astronomical Observatory in Kiev. A preliminary compiled catalogue of similar kind has been constructed at this observatory by Rybka (1990). The GPM catalog will be an updated version based on more comprehensive data from individual catalogues and will consist of two parts. At present, the first part of the GPM catalogue (GPM1) has been completed for the brighter stars common with the HIPPARCOS Input Catalogue (HIC). The second part will contain all remaining stars with limiting photographic magnitude $15.5^{\mathrm{m}}$. Some information about the photographic observations used for deriving the proper motions is given in Table 1 . The observations have been carried out by means of normal (Pulkovo, Tashkent) and long-focus (Kiev, Moscow, Shanghai) astrographs.

Proper motions have been determined from plate measurements. To derive them, 1 to 5 pairs of plates with epoch differences from 20 to 40 years were used. As a 
rule, 40 - 90 faint anonymous stars in the magnitude range $13^{\mathrm{m}}-15^{\mathrm{m}}$ were selected as reference stars to obtain relative proper motions. The relative proper motions were then transformed to an absolute reference frame using galaxies in each field of sky. Practically, the zeropoint calibrations were simply equal and opposite to the mean relative proper motions of several galaxies. The list of centre coordinates of 157 areas with galaxies in the Northern hemisphere (Deutch et al. 1955) and 46 areas in the Southern one (Fatchichin et al. 1959) was compiled at Pulkovo and Tashkent observatories. It contains all available galaxies in KSZ fields. The galaxies were checked to be suitable for precise measurements in terms of 10 number scale.

Table 1. Information about observations

\begin{tabular}{lcccc} 
Observatory & $\begin{array}{c}\text { Zones } \\
\text { degrees }\end{array}$ & $\begin{array}{c}\text { Latitude } \\
\text { degrees }\end{array}$ & $\begin{array}{c}\text { Focus, } \\
\mathrm{m}\end{array}$ & $\begin{array}{c}\text { Number } \\
\text { of fields }\end{array}$ \\
\hline Pulkovo & $+90 \div-5$ & +60 & 3.5 & 89 \\
Tashkent & $+90 \div-25$ & +41 & 3.5 & 157 \\
Kiev & $+90 \div-15$ & +50 & 5.5 & 136 \\
Moscow & $+90 \div-10$ & +55 & 6.4 & 10 \\
Shanghai & $+90 \div-25$ & +31 & 6.9 & 8 \\
\hline
\end{tabular}

The proper motion data were extracted from nearly 30 publications issued since 1952 . The complete list of initial catalogues, explanation of their abbreviations, bibliographic references and some statistics are being prepared by Rybka (1995) for publication. Finally, about 80000 stars from ten individual catalogues have been included in the database. It consists of 185 fields with 550 galaxies nearly uniformly distributed over the sky north of declination -25 degrees outside the Milky Way. The size of each field is nearly 1.5 square degrees. Complete verification of the data, both manually and by computer, has been performed.

\section{Representation of systematic differences and construction of common system}

The construction of the proper motion system of the compiled catalogue was carried out in the usual way. Systematic differences needed for this purpose have been derived through pair-wise comparison of individual catalogues. Preliminary, magnitude dependent errors of proper motions were redetermined for each initial catalogue. Usual statistical methods were applied to this purpose. In such case systematic differences do not depend on brightness of stars and become less than 3 mas/yr.

The investigation of systematic differences has been performed by the analytic method developed at
Heidelberg (Schwan 1988). The systematic relations between catalogues were represented by a series development using orthogonal functions. The orthogonal functions adopted in this work are the products of Legendre polynomials, Hermite polynomials and Fourier terms. Using the transformed declination this type of functions is the most suited for modelling the systematic differences between catalogues under consideration. Only the significant terms of the series development were actually used. The F-test with the level of five percent was used for this purpose. The analysis shows that systematic differences between catalogues depend on right ascension and declination and do not depend on magnitude. The significant terms and their rms errors were applied for deriving a common system of proper motions. In order to do it we were guided by the idea that the weighted mean system is the most reliable one. The weights of catalogues have been assigned according to their consistency with the mean system. The proper motions of initial catalogues have been reduced to the adopted system by applying quite small corrections, with values less than 4 mas/yr.

After the proper motion differences had been corrected for the systematic part, we investigated the random errors in each initial catalogue. It was found that the mean errors are different for individual catalogues with values between 4 and $12 \mathrm{mas} / \mathrm{yr}$. The weighted mean proper motions were calculated for common stars using the weights assigned to each catalogue as the inverse square of the random errors.

\section{HIC stars in GPM1}

Using the results of the above analysis, the compiled catalogue of absolute proper motions for a sample of HIPPARCOS stars was derived from individual catalogues. The sample includes all available HIPPARCOS stars in the selected sky fields with galaxies. The compiled catalogue comprises absolute proper motions for 977 stars in 180 areas north of -25 degrees of declination. The mean square error is $8 \mathrm{mas} / \mathrm{yr}$ on average. The standard errors differ from one star to another one depending on the number and quality of individual catalogues. They have been computed and presented for each star. For a single GPM1 field the absolute zero-point error of linking proper motions to the galaxies is $3 \mathrm{mas} / \mathrm{yr}$ on average. The error of such a kind depends on the number and compactness of the galaxies available in each field. This information may be found elsewhere (Deutch 1955; Fatchikhin 1959). In addition to absolute proper motions and their mean errors, the catalogue gives the following data for each star: right ascension, declination, epoch for position, $V$-magnitude and number, copied from HIC. The stars were also identified with the objects of the Smithsonian Astrophysical Observatory Star Catalogue (SAO) list. Positions are given for equator and equinox J2000.0 on the system of FK5. They were presented to 
Table 2. Example of the GPM1 catalogue

\begin{tabular}{|c|c|c|c|c|c|c|c|c|c|c|}
\hline$\overline{\text { GPM1 }}$ & $\begin{array}{r}\text { KSZ or } \\
\text { NGC }\end{array}$ & $\mathrm{HIC}$ & $\bar{V}$ & $\alpha_{J 2000}$ & $\delta_{J 2000}$ & Ep & $\mu_{\alpha} \cos \delta$ & $\mu_{\delta}$ & $\sigma_{\mu}$ & $\mathrm{SAO}$ \\
\hline 256 & 46 & 43086 & 9.145 & 084633.266 & +184539.14 & & -30.8 & -7.7 & 7.0 & 98114 \\
\hline 257 & 46 & 43199 & 10.500 & 084801.773 & +184037.65 & 87 & -39.8 & -7.9 & 5.6 & \\
\hline 258 & 48 & 43311 & 8.390 & 084923.825 & +504026.05 & & -30.3 & +19.5 & 7.0 & 27006 \\
\hline 259 & 48 & 43339 & 9.100 & 084945.142 & +512914.18 & & +0.6 & -28.6 & 7.0 & \\
\hline 260 & 46 & 43540 & 8.500 & 085203.537 & +185339.03 & & -16.1 & -18.1 & 10.4 & 98182 \\
\hline 261 & 46 & 43564 & 8.498 & 085219.993 & +192102.45 & & -25.0 & +5.7 & 10.4 & 98186 \\
\hline 262 & 48 & 43632 & 9.300 & 085308.762 & +502242.12 & & -9.5 & +3.5 & 7.0 & 27029 \\
\hline 263 & 49 & 43945 & 10.100 & 085702.663 & +025427.14 & & +2.0 & +7.6 & 8.9 & 117289 \\
\hline 264 & 48 & 43962 & 7.200 & 085711.731 & +504430.32 & & -33.5 & -29.3 & 7.0 & 27058 \\
\hline 265 & 49 & 43966 & 6.950 & 085713.635 & +023129.39 & & -44.9 & +7.1 & 8.9 & 117292 \\
\hline 266 & 49 & 44016 & 9.100 & 085748.684 & +032607.81 & & -17.3 & -20.2 & 8.9 & 117306 \\
\hline 267 & 48 & 44023 & 8.770 & 085755.518 & +510913.34 & & +31.3 & +7.8 & 7.0 & 27060 \\
\hline 268 & 48 & 44032 & 8.877 & 085758.934 & +512547.83 & & +90.0 & -130.1 & 7.0 & 27062 \\
\hline 269 & 48 & 44073 & 8.494 & 085842.272 & +520011.80 & & -1.6 & -12.3 & 7.0 & 27066 \\
\hline 270 & 49 & 44105 & 7.992 & 085859.324 & +033921.89 & & -12.6 & +2.2 & 8.9 & 117318 \\
\hline 271 & 48 & 44144 & 8.600 & 085924.637 & +511025.11 & & -47.9 & -12.9 & 7.0 & 27072 \\
\hline 272 & 48 & 44252 & 8.570 & 090044.383 & +505543.94 & & -8.4 & -17.3 & 7.0 & 27082 \\
\hline 273 & 49 & 44363 & 8.100 & 090204.730 & +031647.11 & & -18.7 & -12.7 & 8.9 & 117364 \\
\hline 274 & 53 & 44758 & 8.500 & 090719.478 & +451032.82 & & -88.3 & -50.3 & 10.4 & 42700 \\
\hline 275 & 172 & 44860 & 7.291 & 090825.225 & -150841.22 & & -508.2 & -198.9 & 6.7 & 154939 \\
\hline 276 & 173 & 44881 & 8.800 & 090841.247 & -235304.40 & & -31.9 & -1.8 & 10.4 & 177019 \\
\hline 277 & 53 & 44913 & 8.800 & 090900.420 & +440311.35 & & -36.9 & +4.5 & 10.4 & 42719 \\
\hline 278 & 53 & 44947 & 10.600 & 090921.978 & +444417.34 & & -2.8 & -14.4 & 10.4 & \\
\hline 279 & 172 & 45039 & 7.814 & 091024.810 & -145400.60 & & -8.0 & -20.4 & 6.7 & 154973 \\
\hline 280 & 172 & 45102 & 8.000 & 091117.800 & -145651.50 & & -14.0 & -41.4 & 6.7 & 154982 \\
\hline 281 & 53 & 45160 & 8.500 & 091200.697 & +453032.72 & & +41.1 & -2.0 & 10.4 & 42738 \\
\hline 282 & 53 & 45224 & 8.563 & 091258.210 & +453930.53 & & -3.8 & +0.7 & 10.4 & 42747 \\
\hline 283 & 173 & 45245 & 7.700 & 091320.734 & -243735.76 & & -5.5 & -25.8 & 10.4 & 177126 \\
\hline 284 & 53 & 45294 & 10.100 & 091349.485 & +444324.47 & & -11.2 & -29.4 & 10.4 & \\
\hline 285 & 53 & 45348 & 9.300 & 091427.590 & +445247.70 & & -18.3 & -15.8 & 10.4 & 42761 \\
\hline 286 & 53 & 45360 & 9.100 & 091436.414 & +452559.88 & & +19.4 & -72.7 & 10.4 & 42764 \\
\hline 287 & 173 & 45364 & 8.838 & 091441.696 & -235704.43 & & -12.8 & -25.1 & 10.4 & 177161 \\
\hline 288 & 53 & 45401 & 9.000 & 091508.048 & +440259.12 & & +28.9 & -286.2 & 10.4 & 42766 \\
\hline 289 & 53 & 45447 & 9.500 & 091543.832 & +451737.75 & 87 & -16.1 & -53.9 & 10.4 & \\
\hline 290 & 53 & 45450 & 9.400 & 091546.202 & +441523.35 & & +14.3 & -10.5 & 10.4 & 42770 \\
\hline
\end{tabular}

identify the GPM1 stars with those from other catalogues. The data file is sorted by $\mathrm{J} 2000.0$ right ascensions.

A sample page from the GPM1 catalogue is given in Table 2. A brief description of the columns of Table 2 follows:

Column 1: GPM1 running number.

Column 2: Region number. Numbers from 1 to 205 are given according to the KSZ plan, numbers larger than 205 are NGC numbers of centre galaxies.

Column 3: HIC number.

Column 4: $V$ magnitude, copied from HIC.

Column 5: The right ascension, $\alpha$, is given for equinox J2000.0, in hours, minutes, and seconds, copied from HIC.

Column 6: The declination, $\delta$, is given for equinox J2000.0, in degrees, minutes, and seconds, copied from HIC.
Column 7: The epoch for the position, Ep: blank for J2000.0; otherwise (Epoch-1900), copied from HIC.

Column 8: Absolute proper motion in right ascension, $\mu_{\alpha} \cos \delta$, is expressed in mas/yr.

Column 9: Absolute proper motion in declination, $\mu_{\delta}$, is expressed in mas/yr.

Column 10: Mean error of proper motion, $\sigma_{\mu}$, is expressed in mas/yr.

Column 11: SAO number.

\section{Comparison between the GPM1 and other catalogues}

To test the validity of the new proper motions from the GPM1, we have carried out comparison with those from the PPM (Roeser \& Bastian 1993) and ACRS (Corbin \& Urban 1990), determined both on the FK5 system. It 
also allows us to estimate the value of the angular vector velocity originating from spurious rotation of the FK5 system, because proper motions referred to galaxies are assumed to be in a nonrotating system. The proper motion differences PPM-GPM1, ACRS-GPM1 needed for this purpose have been computed for common stars. 837 stars are common with PPM and 743 with ACRS catalogues. The systematic relations between the above catalogues have been derived by least square fits using the known equations, see for instance (Brosche et al. 1995):

$$
\begin{aligned}
& \Delta \mu_{\alpha} \cos \delta=-\omega_{1} \cos \alpha \sin \delta-\omega_{2} \sin \alpha \sin \delta+\omega_{3} \cos \delta \\
& \Delta \mu_{\delta}=\omega_{1} \sin \alpha-\omega_{2} \cos \alpha .
\end{aligned}
$$

Here $\alpha, \delta$ are star equatorial coordinates, $\Delta \mu_{\alpha} \cos \delta$, $\Delta \mu_{\delta}$ are the proper motion differences between PPM (or ACRS) and GPM1 in right ascension and declination respectively, while $\omega_{i}$ are the components of the rotation vector. In the relations under consideration the two reference systems are assumed to differ by a rigid rotation, neglecting any kind of systematic errors that may be present in either system. The results that have been found from combined $\Delta \mu_{\alpha} \cos \delta$ and $\Delta \mu_{\delta}$ solution are given in Table 3 , where $\epsilon$ denotes the standard errors of the unknowns $\omega_{i}$.

Table 3. The components of rotation vector

\begin{tabular}{lccc}
\hline \multirow{2}{*}{ Catalogue } & $\omega_{1} \pm \epsilon$ & $\omega_{2} \pm \epsilon$ & $\omega_{3} \pm \epsilon$ \\
& $\mathrm{mas} / \mathrm{yr}$ & $\mathrm{mas} / \mathrm{yr}$ & $\mathrm{mas} / \mathrm{yr}$ \\
\hline PPM-GPM1 & $+1.5 \pm 0.8$ & $+1.2 \pm 0.6$ & $-2.5 \pm 0.6$ \\
ACRS-GPM1 & $+1.2 \pm 0.8$ & $+0.9 \pm 0.7$ & $-1.7 \pm 0.6$ \\
\hline
\end{tabular}

Table 3 shows that we have significant values $\omega_{i}$ from the above solution. Therefore, considering that the accuracy of HIPPARCOS proper motions is higher than in the PPM or ACRS, the GPM1 contains a sufficient number of stars to yield statistically significant values of the rotation of the HIPPARCOS system.

\section{Conclusions}

The current phase of the GPM programme has now culminated in the GPM1 catalogue for a sample of HIPPARCOS stars. The GPM1 originates directly from the data reduction of independent initial catalogues containing proper motions referred to galaxies. It may be expected that by combining stars into a compiled catalogue the accuracy of the absolute proper motions will be improved with regard to both random and systematic errors. The first application of the catalogue is the study of rotation of the FK5 system, as reported here. It has been based on a comparison with the PPM and ACRS proper motions. The investigation of proper motion differences PPM-GPM1 and ACRS-GPM1 has shown that the residual rotation of the FK5 system is less than 2 mas/yr. Thus it should be noted that the data from the GPM1 may be used for transferring the HIPPARCOS proper motions to an absolute reference frame. At present the final GPM catalogue is in preparation. It will include proper motions of over 60000 stars, covering the $B$-magnitude range $8^{\mathrm{m}}-15.5^{\mathrm{m}}$.

Acknowledgements. A long term astrometric programme of such kind as GPM involves several generations of astronomers. To all of them we give our deepest acknowledgement and profound thanks. The authors acknowledge support provided by grant from the ESO. We should like to thank all collaborators participating in programme "HIPPARCOS extragalactic link by photographic astrometry". Our particular thanks we express to U. Bastian, S. Roeser, C. Turon and V. Kislyuk for fruitful discussion of the manuscript.

\section{References}

Brosche P., et al., 1995, HIPPARCOS extragalactic link. In: Hog E., Seidelman P.K. (eds.) Astronomical and astrophysical objectives of sub-milliarcsecond optical astrometry. Kluwer, Dordrecht / Boston / London, p. 380

Corbin T.E., Urban S.E., 1990, Faint reference system. In: Lieske J.H., Abalakin V.K. (eds.) Inertial coordinate system on the sky. Kluwer, Dordrecht /Boston / London, p. 433

Deutch A.N., 1952, Trans. IAU 8, 789

Deutch A.N., Lavdovsky V.V., Fatchikhin N.V., 1955, Izvestija GAO Pulkove 20, 1

Fatchikhin N.V., Latypov A.A., 1959, Tsirkuljar Tashkent Astron. Observ. 302, 3

Kharchenko N.V., 1987, Kinematikya Fiz. Nebesn. Tel 3, 7

Roeser S., Bastian U., 1993, Bull. Inform. CDS 42, 11

Rybka S.P., 1990, Kinematika Fiz. Nebesn. Tel 6, 54

Rybka S.P., 1995, Kinematika Fiz. Nebesn. Tel (in press)

Schwan H., 1988, A\&A 198, 363

Yatsenko A.I., Rybka S.P., Scholz R.-D., 1987, Astron. Nachr. 308,319 